\title{
Neumomediastino espontáneo
}

\section{Reporte de un caso}

García Muñoz I*, Rocha Peña E*, Torres Escamilla J*

Flores Álvarez E**, Gallegos Ortega José de J***

\section{Introducción}

El neumomediastino es una entidad reportada inicialmente por Hamman en 1939 y su fisiopatogenia descrita por Macklin en 1944. Se define como la presencia anormal de aire en el mediastino de cualquier etiología. El neumomediastino espontáneo es una enfermedad benigna, infrecuente, autolimitada, que se ha asociado a la inhalación de cocaína ${ }^{1}$.

El neumomediastino es una complicación que se presenta hasta en el $10 \%$ de los enfermos con trauma cerrado de tórax; el gas puede disecar las vainas músculo-aponeuróticas y extenderse al cuello o a la cavidad abdominal llegando a ocasionar neumoperitoneo ${ }^{2}$. Generalmente es ocasionado por una lesión o desgarro tráqueo-bronquial o esofágico y en caso de neumoperitoneo a perforación de una víscera hueca abdominal.
Cuando se descarta la existencia de una lesión primaria se considera que fue ocasionado por la ruptura de alvéolos en el parénquima pulmonar con diseminación del gas hacia el mediastino por un episodio agudo de elevación de la presión intratorácica, fenómeno conocido como efecto Macklin ${ }^{3}$.

El neumomediastino espontáneo ha sido reportado predominantemente en varones jóvenes. Representa una entidad poco frecuente, con una incidencia reportada de 1 por cada 10.000 ingresos hospitalarios y representa el $1 \%$ del total de casos de neumomediastino ${ }^{4}$.

El objetivo del trabajo es reportar un caso de neumomediastino secundario al efecto Macklin en un paciente consumidor de cocaína.

\section{Presentación del caso}

Se trata de un paciente masculino de 16 años de edad que se presentó al servicio de urgencias del Centenario Hospital Miguel Hidalgo con un cuadro de 12 horas de evolución caracterizado por disnea de aparición súbita y dolor torácico de tipo punzante,

* Medico Residente de Cirugía General.

** Cirujano General. Cirujano Oncólogo. Maestro en Ciencias. Profesor Titular Posgrado Cirugía General.

*** Cirujano General.

**** Otorrinolaringólogo.

Departamentos de Enseñanza y Cirugía, Centenario Hospital Miguel Hidalgo, ISEA. 
de localización retroesternal, acompañado de náusea y malestar general, ocurrido después de la inhalación impulsiva de cocaína en repetidas ocasiones.

A su ingreso al servicio de urgencias se le encontró con estabilidad hemodinámica, agitación psicomotriz, polipneico, con enfisema subcutáneo que se extendía sobre ambos lados de la cara anterior y lateral del cuello y el tercio superior de la cara anterior de la pared costal (figura 1). No se detectó ninguna anormalidad en el precordio, tampoco se identificaron datos de alteración cardiovascular ni se integró algún síndrome pleuro-pulmonar.

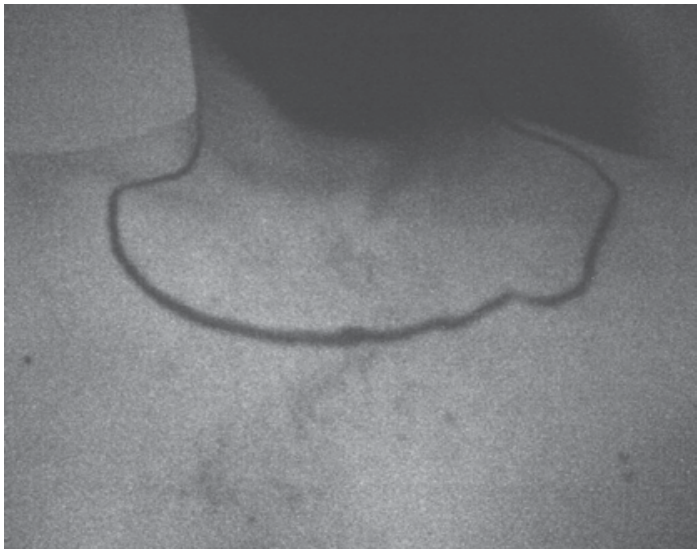

La telerradiografía de tórax mostró una imagen radiolúcida delimitando el contorno cardiaco izquierdo con extensión por el mediastino hasta el cuello, descartó la existencia de fracturas de arcos costales y la presencia de líquido en los senos costofrénicos o cardiofrénicos (figura 2 y 3 ).

Figura 1: Extensión del enfisema subcutáneo en el cuello y la cara anterior del tórax.

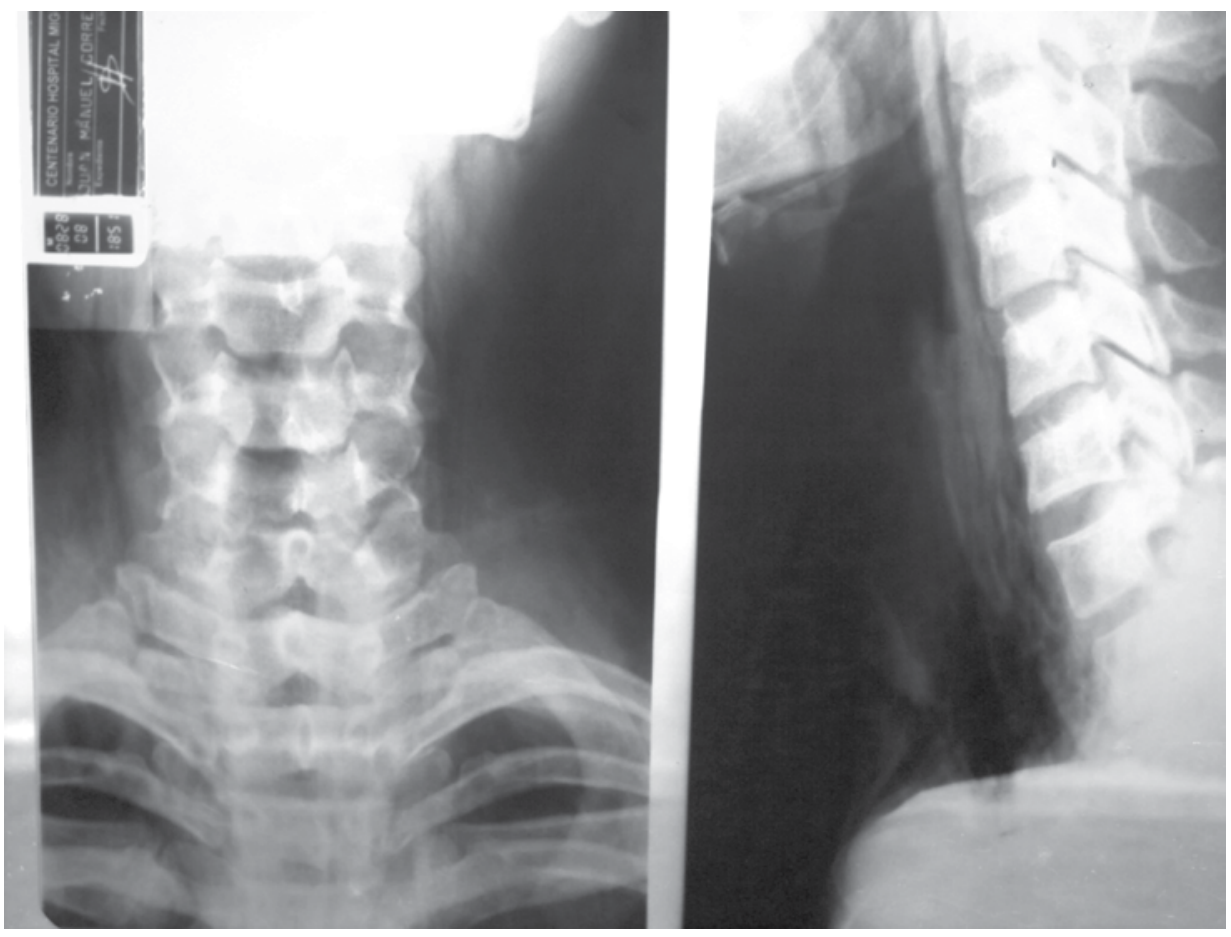

Figura 2: Radiografía anteroposterior y lateral del cuello que muestran la presencia de gas subcutáneo. 


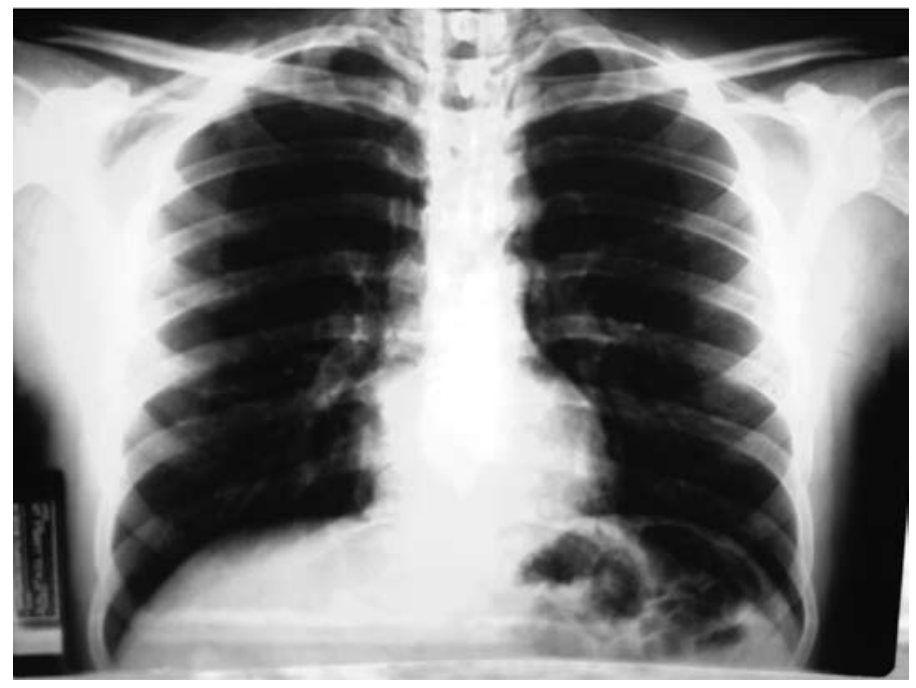

En la tomografía computada de cuello y tórax se observó la presencia anormal de gas en las fascias de los tejidos blandos del cuello y mediastino. Se descartó la presencia de lesiones pleuro-pulmonares; la pared esofágica y el resto de las estructuras mediastinales se encontraron intactas (figura 4 y 5).

Figura 3: Telerradiografía de tórax que evidencía la presencia de neumomediastino.

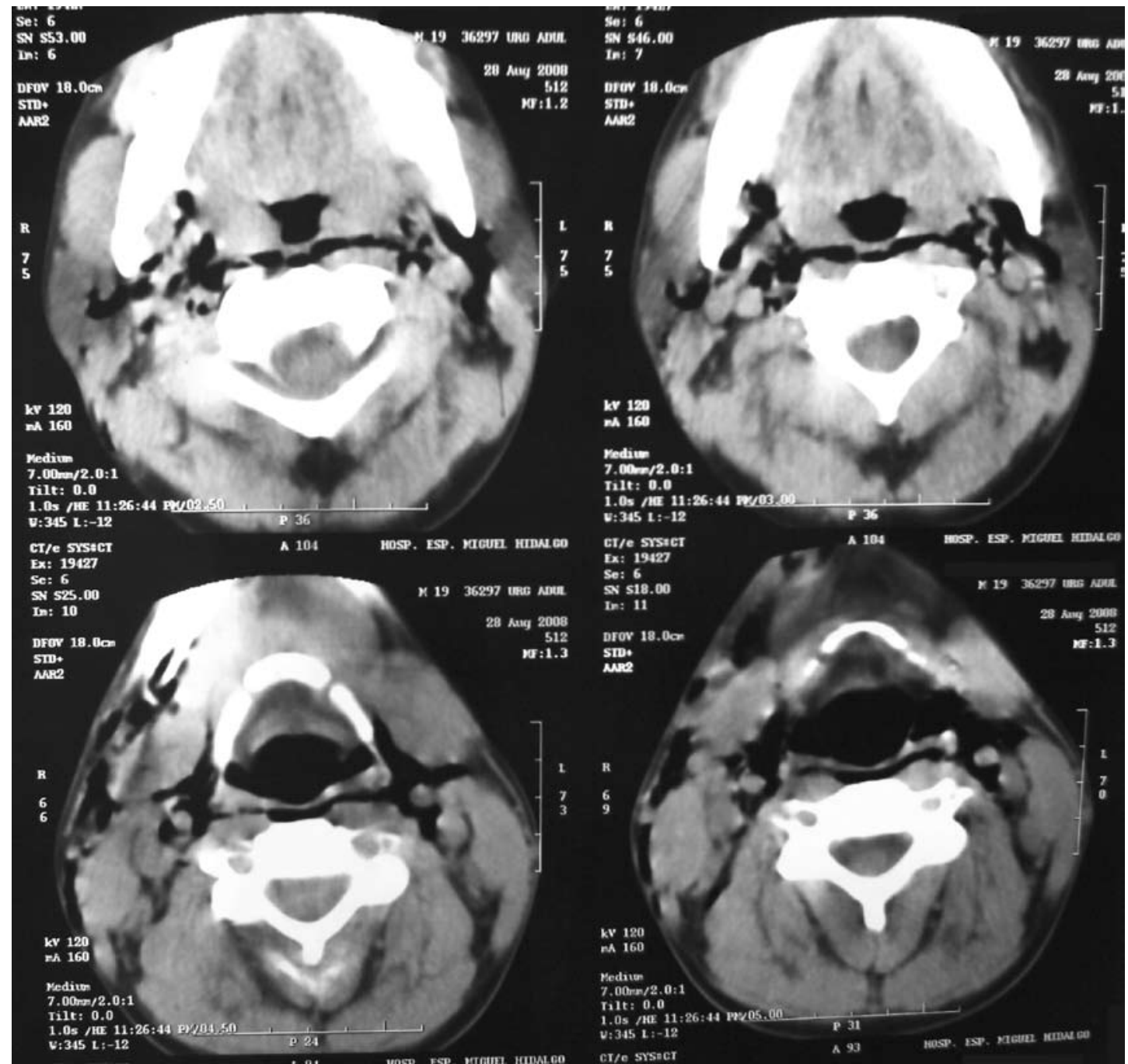

Figura 4: Tomografía computada de cuello. Muestra gas que diseca las fascias profundas del cuello. 


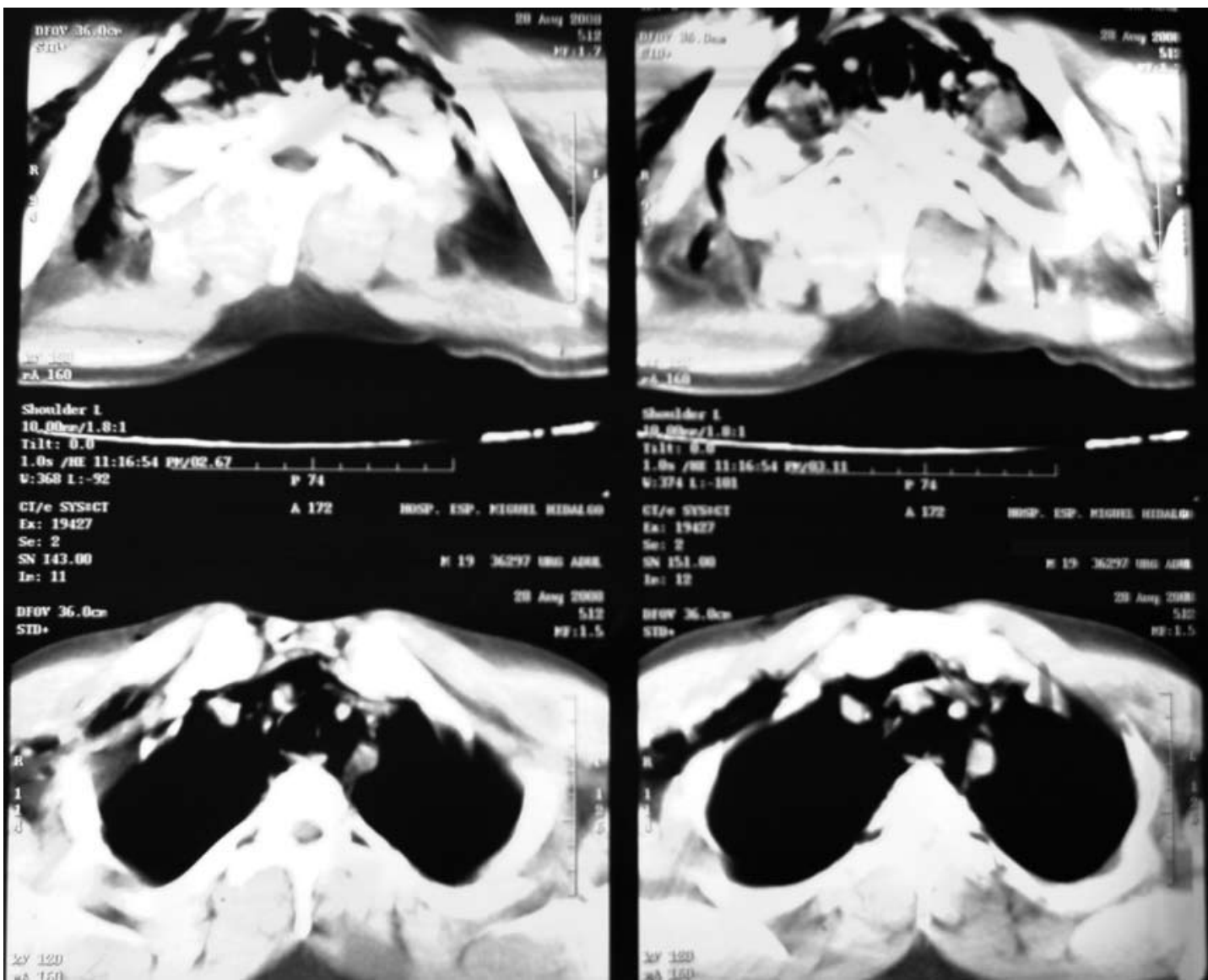

Figura 5: Tomografía de Tórax que corrobora la presencia de neumomediastino.

Por medio de ecocardiografía se descartaron lesiones pericárdicas, alteraciones valvulares y miocárdicas; se encontró una fracción de expulsión y diástole normales.

Por broncoscopia se observó un árbol tráqueo-bronquial sin lesiones y la endoscopia del tubo digestivo superior descartó alteraciones a nivel esofágico-gástrico. Al descartarse una lesión primaria como causa del cuadro clínico se consideró que se trataba de un neumomediastino secundario a un efecto Macklin.

El paciente fue manejado en forma conservadora mediante oxígeno suplementario y estricta monitorización cardiovascular por parte de los servicios de cirugía general, otorrinolaringología, cirugía cardiotorácica y neumología. Se administraron analgésicos y antibiótico profiláctico. Se mantuvo en reposo intestinal por 5 días. La evolu- ción fue favorable sin desarrollar complicaciones a ningún nivel y fue egresado a los 10 días.

\section{Discusión}

La presencia de fuga aérea hacia el mediastino se asocia a lesiones traumáticas graves del esófago o del aparato respiratorio que de no ser diagnosticadas y tratadas de manera temprana y correcta se asocian a una elevada morbilidad y mortalidad. En aquellos pacientes en los que no se encuentra la causa de la fuga aérea después de una evaluación exhaustiva de la integridad del esófago, el árbol tráqueo-bronquial y del espacio pleural se considera que se trata de un neumotórax espontáneo secundario a un efecto Macklin ${ }^{5}$.

El efecto Macklin fue descrito en 1939 por el Dr. Charles Macklin y explica la etio- 
logía de la fuga aérea hasta en 39\% de los enfermos con trauma cerrado de tórax. Se ha descrito también en el síndrome de insuficiencia respiratoria del recién nacido, en la ventilación mecánica con presión positiva y con gradientes transpleurales elevados, en crisis asmática, maniobras de Valsalva y en la hiperinflación dinámica ${ }^{6}$.

El neumomediastino espontáneo se manifiesta clínicamente con sensación de opresión retroesternal, disnea, disfagia y disfonía. El enfisema subcutáneo es el signo clínico más comúnmente identificado. En la radiografía simple de tórax se evidencía la presencia de gas en el mediastino. En el estudio de frente se observa un halo radiolúcido contorneando la silueta cardiaca y en ocasiones es posible identificar gas entre las vainas peribroncovasculares. En el enfoque lateral se puede visualizar gas en el espacio subcutáneo y retroesternal ${ }^{7}$. La tomografía permite descartar la existencia de una enfermedad pulmonar de base o un neumotórax asociado ${ }^{8}$.

La causa directa del neumomediastino espontáneo es desconocida. Su presentación se ha relacionado a múltiples esfuerzos, episodios de tos severa, vómitos intempestivos y aspiración de cuerpos extraños. El mecanismo etiopatogénico descrito es el aumento súbito de la presión intraalveolar asociada a una espiración vigorosa con la glotis cerrada (maniobra de Valsalva). Cuando ésta excede la presión de la vascularización pulmonar se produce la ruptura alveolar en su base, dando salida a aire ocasionando enfisema intersticial que diseca las vainas peribroncovasculares y los septos interlobares. Por gradiente de presión el aire puede progresar hacia los hilios y producir neumomediastino y neumotórax o bien avanzar hacia la periferia pulmonar y originar burbujas subpleurales o neumotórax. El aire puede diseminarse hacia los tejidos subcutáneos de la pared torácica y del cuello dando origen al enfi- sema subcutáneo detectado en la exploración física ${ }^{8,9,10}$.

El uso de cocaína o sus derivados pasta base como droga ha ido en aumento en los últimos años. La pasta base o base de coca es el derivado más barato, contaminante y tóxico de la cocaína. Su uso por vía inhalatoria se ha relacionado con complicaciones cardiovasculares y pulmonares, incluidos el neumomediastino, el neumotórax y el neumoperitoneo. El neumomediastino se asocia con mayor frecuencia a la inhalación de vapores de cocaína en porcentajes variables que fluctúan entre el $2,8 \%$ y el $75 \%{ }^{11}$.

La inhalación de la droga en pacientes que desarrollan neumotórax espontáneo está relacionada con el consumo compulsivo con esfuerzos prolongados, progresivos y profundos, seguidos de la maniobra de Valsalva. Además del barotrauma, se postula un efecto vasoconstrictor de la cocaína sobre los vasos alveolares que induce la ulterior rotura alveolar y paso del aire al mediastino. Algunos autores sugieren como mecanismo secundario la existencia de una alteración del surfactante y la elasticidad pulmonar ${ }^{12,13}$.

En general, los pacientes evolucionan favorablemente con el manejo conservador. Las medidas incluyen el reposo absoluto en cama, ayuno con apoyo nutricional con dieta enteral o parenteral, fluidoterapia, oxígeno suplementario, analgésicos y fisioterapia pulmonar. Se han reportado casos de pacientes con neumomediastino tan amplio que puede llegar a ocasionar compresión venosa con descenso de la presión arterial al generarse una caída del retorno venoso a las cavidades cardíacas derechas ${ }^{10,15}$.

También se ha observado que el consumo continuado de cocaína puede ocasionar episodios recurrentes, cada uno de los cuales empeora gradualmente el pronóstico de los pacientes ${ }^{13}$. 


\section{Conclinsiones}

Siempre debe considerarse el diagnóstico de neumotórax espontáneo en pacientes con dolor torácico y disnea, particularmente en consumidores de drogas inhaladas. Debe establecerse un manejo conservador con estricta monitorización hemodinámica y control radiográfico hasta la resolución del cuadro.

\section{Bibliografía}

1 D'urbano C, Fuertes GF, Biraghi T. Pneumomediastinum: physiopathological considerations and report of six cases treated in an emergency surgery division. Minerva Chir 1996;51:577-583.

2 Cyrlak D, Milne ENC, Imray TJ. Pneumomediastinum: a diagnostic problem. Crit Rev Diagn Imaging 1984;23: 75-79.

3 Wintermark $M$, Wicky S, Schnyder P. Blunt traumatic pneumomediastinum: using CT to reveal the Macklin effect. AJR 1999;172:129-130.

4 Wintermark $M$, Schnyder. The Macklin effect: A frequent etiology for pneumomediastinum in severe blunt chest trauma. Chest 2001;120:543-547

5 Pilar B, Arce J. Neumomediastino espontáneo: enfisema retrofaringeo forma de presentación no habitual. Rev Chil Radiol 2005; 3(11): 116-21.

6 De La Cruz Morón I, Reyes Nuñez N, Rojas Box JL. Neumomediastino espontáneo en un consumidor de cocaina. Arch Bronconeumol 2000; 36(4): 231.

7 Santiago Aguinaga IJ, Martínez-Bayarri M. Neumomediastino espontáneo: análisis de 16 casos. Emergencias 2000; 12: 321-5.

8 Rubio J, Bioque J, Feu N, Martínez Pacual, García F, Muñoz. Neumomediastino en un fumador libre de pasta de cocaina. Servicio de Neumologia del Hospital Universitario Reina Sofía, Córdoba. España.
9 Gerazounis M, Athanassiadi K, Kalantazi N, Moustordas $M$. Spontaneous pneumomediastinum: a rare benign entity. J Thorac Cardivasc Surg 2003; 126(3): 774-6

10 Androuny A, Magnusson P. Pneumopericardium from cocaine inhalation. N Engl J Med 1985; 313(1): 48-9.

11 Shesser R, Davis C, Edelstein S. Pneumomediastinun and pneumothorax after inhaling alkaloidal cocaine. Ann Emerg Med 1981; 10(4): 213-5.

12 Koullias GJ, Korkolis DP, Wang XJ, Hammond GL. Current assessment and management of spontaneous pneumomediastinum in 24 adult patients. Eur J Cardiothorac Surg2004; 25(5): 852-5.

13 Rello J, Net A. Complicaciones asociadas al consumo de cocaina. Med Clin (Barc) 1988; 91(11): 435

14 Aroesky DJ, Stanley RB, Crockett DM. Pneumomediastinum and cervical emphysema from inhalation of .free based. cocaine: report of three cases. Otolaryngol Head Neck Surg 1986; 94(3): 372-4.

15 Langwieler TE, Steffani KD, Bogoesvki DP, Mann O, Izbicki JR. Spontaneous pneumomediastinum. Ann Thorac Surg 2004; 78(2): 711-3 